\title{
Ultrafast Double Potential Step Chronoamperometry with the Time Window Down to Submicrosecond
}

\author{
Xiangqin LIN` and Zhiyong Guo \\ Department of Chemistry, University of Science and Technology of China, Hefei 230026, P. R. China
}

\begin{abstract}
Ultrafast double potential step chronoamperometry at an ultramicroelectrode was achieved through decreasing the ohmic drop by positive feedback compensation, and the lower limit of the time window was extended to the submicrosecond level. With the reduction of anthracene in acetonitrile as a test system, the validity of this instrumentation was demonstrated by comparing between experimental and simulated curves. The capability in kinetic studies of the technique was then approved.
\end{abstract}

(Received May 13, 2004; Accepted September 13, 2004)

\section{Introduction}

As reversal techniques, cyclic voltammetry (CV) and double potential step chronoamperometry (DPSC) are both effective approaches to studying electrochemically generated short-lived species. ${ }^{1}$ Compared with CV, DPSC presents attractive advantages of avoiding the interference of heterogeneous charge-transfer kinetics and ohmic drop, whose effect should be taken into account to extract the characteristic rate constants of electrogenerated intermediates in CV. ${ }^{1-3}$ Obviously, the more rapid is the scan rate or high frequency employed, the faster are the heterogeneous and homogeneous reactions that may be studied, and shorter-lived electrogenerated species that can be traced. Thus, decreasing the lower limit of the time window of DPSC is an important objective for fast electrochemical studies. However, the reported values ${ }^{2,4,5}$ were high, from several to a few tens of microsecond, which restricted its applications in kinetics. If the instrumentation response is sufficiently quick, the lower limit of the time window lies on the cell time constant, $R_{\mathrm{s}} C_{\mathrm{d}}$, which can be considered to reduce mainly in the following ways: (a) Using ultramicroelectrodes. At a disk ultramicroelectrode, the solution resistance $\left(R_{\mathrm{S}}\right)$ is reciprocal to the electrode radius $(r)$, while the capacitance of the double layer $\left(C_{\mathrm{d}}\right)$ is proportional to the electrode area i.e. $\left(r^{2}\right)$; thus, $R_{\mathrm{s}} C_{\mathrm{d}} \sim r \rightarrow 0$ when $r \rightarrow 0$. (b) Increasing the conductivity of the medium through an increase in the supporting electrolyte concentration or solvent polarity to reduce $R_{\mathrm{s}}$. (c) Moving the reference electrode tip as close as possible to the working electrode so as to reduce the uncompensated resistance $\left(R_{\mathrm{u}}\right)$. However, this way is not practical for an ultramicroelectrode, because its $R_{\mathrm{s}}$ is concentrated in the solution on the surface of the electrode with a thickness of $\pi r / 4 .^{6}$ (d) Employing electronic positive feedback technology to compensate $R_{\mathrm{s}}$ online.

In a previous paper of ours, ${ }^{7}$ we described a single-opamp circuit with positive feedback compensation of the ohmic drop, by which undistorted voltammograms could be recorded at a scan rate of up to $2.67 \mathrm{MV} \mathrm{s}^{-1}$. However, ultrafast DPSC could

† To whom correspondence should be addressed.

E-mail: xqlin@ustc.edu.cn not be achieved directly by the circuit due to two main problems: first, the output voltage at low frequency would be too small to be detected by an oscilloscope because of the inherently low gain of the above-mentioned circuit, ${ }^{7}$ because the current of DPSC decreases with the time, while that of CV does not; second, the stability of the above-mentioned circuit could not afford ultrafast DPSC at a frequency of up to $\mathrm{MHz}$.

In this report, a $5 \mu \mathrm{m}$ ultramicroelectrode was used as the working electrode, a new home-made circuit with online positive feedback was utilized to compensate for $R_{\mathrm{s}}$ as much as possible, and a two-electrode system, instead of a threeelectrode one, was applied to lessen the number of operational amplifiers used, to simplify the circuit and to improve the response speed. Then, the lower limit of the time window of DPSC was decreased down to the submicrosecond level, and could thus be used to study the reaction mechanisms and intermediates of faster reactions.

\section{Experimental}

\section{Reagents and instruments}

Anthracene was purchased from Sigma and recrystallized before use. Tetraethylammonium tetrafluoroborate $\left(\mathrm{NEt}_{4} \mathrm{BF}_{4}\right)$ was commercially available (Aldrich), and was dried at $60^{\circ} \mathrm{C}$ under a vacuum and stored in a vacuum desiccator. Acetonitrile (HPLC quality) was distilled from calcium hydride and then degassed by nitrogen just before use.

The input signals were generated from a Model G305 waveform generator (10 MHz, HUNG CHANG, Korea), which were then passed through the electrochemical cell and a homemade circuit. At last, the $I-t$ curves were recorded with a Tektronix Model TDS220 digitizing oscilloscope with a 100 $\mathrm{MHz}$ band-pass. Data were downloaded and stored in a Pentium IV computer for further analysis.

\section{Cell and circuit}

A two-electrode system was employed, including a gold microdisk electrode as the working electrode, a platinum-wire counter/quasi-reference electrode and a $200 \mu$ l glass cell, which was mounted directly on the printed circuit board (PCB) to keep 


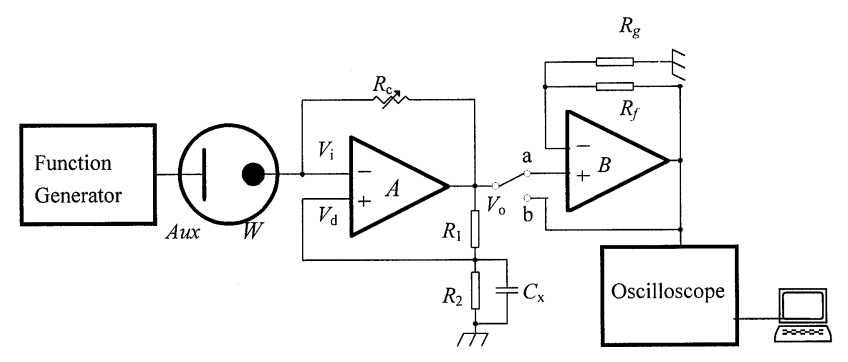

Fig. 1 Scheme of the ultrafast DPSC circuit.

the stray capacitance small and the propagation time short. The $\mathrm{Au}$ microdisk working electrode comprised an $\mathrm{Au}$ wire (Goodfellow Metals, $5 \mu \mathrm{m}$ nominal diameter, purity 99.99\%) sealed in glass. ${ }^{8}$ Just prior to collecting $I-t$ curves each time, it was carefully polished using successively 0.3 and $0.05 \mu \mathrm{m}$ alumina powder ( $\mathrm{CH}$ Instruments, USA) on a clean and damp polishing cloth ( $\mathrm{CH}$ Instruments, USA), and then sonicated continuously in distilled deionized water and acetonitrile.

The scheme of the home-built ultrafast circuit that could compensate $R_{\mathrm{s}}$ online is shown in Fig. 1. Operational amplifier A (MAX4100, Maxim) is actually a negative-impedance current-to-voltage converter. Because the positive feedback voltage $\left(V_{\mathrm{d}}\right)$ produced a negative input impedance, $Z_{\mathrm{i}}=V_{\mathrm{i}} / I \approx$ $V_{\mathrm{d}} / I$, the voltage effectively applied to the electrochemical interface was augmented proportionally to the current. If $Z_{\mathrm{i}}$ could be adjusted by adjusting $R_{\mathrm{c}}$ nicely to cancel out $R_{\mathrm{s}}$, perfect compensation would be achieved. A $10 \mathrm{MHz}$ at $0.1 \mathrm{~dB}$ bandwidth guarantees that it could afford ultrafast electrochemical studies. A thorough theoretical description of its behavior and an experimental demonstration of its validity are described in a related paper. ${ }^{7}$ To maintain the high bandwidth of opamp A, $R_{\mathrm{c}}$ should not be larger than $2 \mathrm{k} \Omega$; thus the output voltage $\left(V_{\mathrm{o}}\right)$ will be too small at low frequency for the oscilloscope. Indeed, it was not a problem for ultrafast $\mathrm{CV}$, because the current of DPSC decreased with the time, while that of $\mathrm{CV}$ did not. In this case, $V_{0}$ was sent through "a" to operational amplifier B (MAX4224, Maxim) and amplified by it on demand. However, at high frequency, $V_{\mathrm{o}}$ was sent to the oscilloscope directly through "b". The circuit described above was designed elaborately on a PCB. The combination of very short electrical leads, lack of switches, surface-mounted electronic components and SMA connecters made its high-frequency performance better. The circuit was placed in a Faraday cage during the experiments.

\section{Results and Discussion}

\section{Determination of the time window}

In DPSC, the potential is first stepped to generate the species of interest, and then stepped back to the original value at a time denoted $\theta$. Kinetic information could be extracted from the current ratio, $i(2 \theta) / i(\theta)$, in which $i(\theta)$ and $i(2 \theta)$ are the current at the times of $\theta$ and $2 \theta$, respectively. When $\theta$ is much smaller than the lifetime of the electrogenerated intermediate, the system becomes chemically reversible, i.e., entirely controlled by the diffusion of the substrate and of its primary reduction product. Then, $[i(2 \theta) / i(\theta)]_{\text {dif }}=1-2^{-1 / 2}$. Thus, the current response could be normalized as $R=[i(2 \theta) / i(\theta)] /[i(2 \theta) / i(\theta)]_{\mathrm{dif}}$, the value of which will be equal to 1 when pure diffusion control is reached. ${ }^{2,3}$

The total current $\left(i_{\mathrm{t}}\right)$ flowing through the electrode is the sum of the Faradaic current $\left(i_{\mathrm{f}}\right)$ and the double-layer charging current
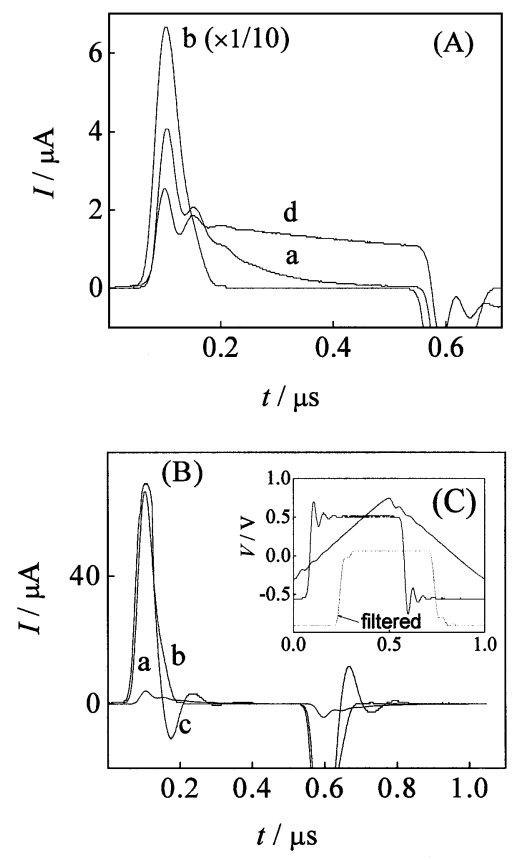

Fig. 2 The lower limit of the time windows at dummy cells of 20 $\mathrm{k} \Omega+4 \mathrm{pF}(\mathrm{a}, \mathrm{b}, \mathrm{c})$ and $50 \mathrm{k} \Omega+10 \mathrm{pF}(\mathrm{d})$ with no compensation (a, d), optimized (b) and "overcompensation" (c).

$\left(i_{\mathrm{c}}\right)$. At very short times after the start of the forward and reversal steps, $i_{\mathrm{c}}$ is the main fraction of $i_{\mathrm{t}}$. However, after the time of $10 R_{\mathrm{s}} C_{\mathrm{d}}$, the contribution of $i_{\mathrm{c}}$ to $i_{\mathrm{t}}$ could be ignored, as $i_{\mathrm{c}}$ decays exponentially with the time constant $R_{\mathrm{s}} C_{\mathrm{d}}$, while $i_{\mathrm{f}}$ is proportional with $t^{-1 / 2}$. Therefore, $10 R_{\mathrm{s}} C_{\mathrm{d}}$ could be adopted tentatively as a lower limit of the time window, which is actually also related to the performance of the whole circuit system. The upper limit of the time window rests on the decrease of the Faradaic current down to the noise level. In our case, it is $20-40 \mu \mathrm{s}$.

With a square wave of $1 \mathrm{~V}$ amplitude and $1 \mathrm{MHz}$ frequency as the input signal, RC dummy cells were used to determine the lower limit of the time window. Figure $2 \mathrm{~A}$ shows that an $i_{\mathrm{c}}$ of $50 \mathrm{k} \Omega+10 \mathrm{pF}$ dummy cell decayed no more than $10 \%$ in 0.5 $\mu \mathrm{s}$, which suggested that the usual experimental condition is not suited for such a kind of ultrafast DPSC analysis at all. Without any ohmic drop compensation, an $i_{\mathrm{c}}$ of $20 \mathrm{k} \Omega+4 \mathrm{pF}$ dummy cell decayed $90-95 \%$ in $0.5 \mu$ s, showing the advantage of using the ultramicroelectrode. If we compensated the ohmic drop at a level of $70 \%$, the $i_{\mathrm{c}}$ of a $20 \mathrm{k} \Omega+4 \mathrm{pF}$ dummy cell decayed to zero in $120 \mathrm{~ns}$ (the output signal (b) was attenuated 10 times for an easy comparison), suggesting that online ohmic drop compensation could lower the time window effectively. Obviously, the higher is the compensation level, the lower is the time window. Unfortunately, the compensation level could not be higher than $70 \%$ (optimized); otherwise, an oscillation would occur once "overcompensation" was reached, as shown in Fig. 2B. This resulted from the $10 \mathrm{~ns}$ time lag of the feedback signal behind the input signal, which would cause some overcorrection to the input signal, and thus overshoot and ringing ensued. However, in our previous studies using a triangular wave as an input signal, ${ }^{7}$ a $95 \%$ compensation level could be reached without any obvious oscillation. The difference was caused by the oscillation of the input signal at the switch point of the potential scan, which was more serious in the case of a triangular wave, as shown in Fig. 2C. This kind of oscillation 

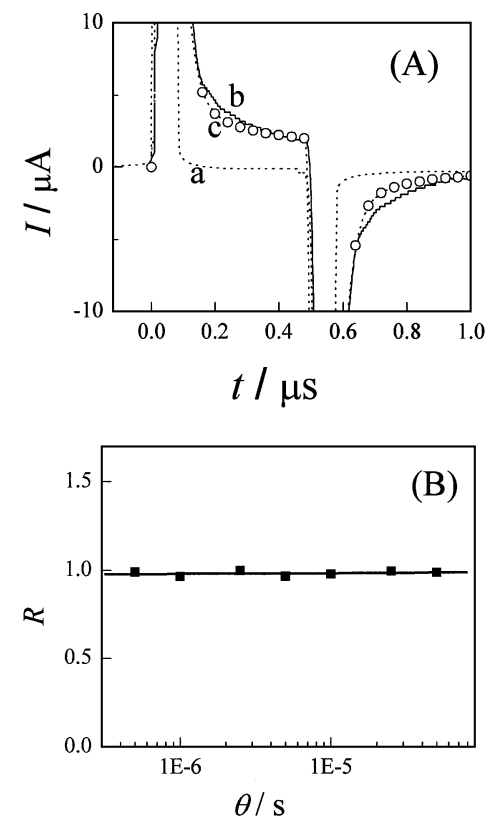

Fig. 3 Ultrafast DPSC of the reduction of anthracene $(15 \mathrm{mM})$ in acetonitrile/0.9 $\mathrm{M} \mathrm{NEt}_{4} \mathrm{BF}_{4}$ at a gold disk of $5 \mu \mathrm{m}$ diameter. Potential was stepped between -1.1 and $-2.2 \mathrm{~V} v s$. Pt electrode. (A) $I-t$ curves $(1 \mathrm{MHz})$ in the presence (a, solid line) and absence (b, dotted line) of anthracene, and the simulated (c, open circles). (B) The plot of current ratio $(R)$ as a function of $\theta$.

produced "overcompensation" ahead. To decrease this oscillation, a $20 \mathrm{~ns}$ low-pass filter could be used without significantly changing the waveform of the input signal. Obviously, tests using dummy cells indicated that the lower limit of the time window of an ultrafast DPSC could reach the submicrosecond level by using this instrumentation.

\section{Reduction of anthracene in acetonitrile as a test system}

As the fastest charge-transfer reaction known, the reduction of anthracene in acetonitrile is typically a chemically reversible system. Thus, throughout the studied time window, $R$ was identical to 1 , and could act as a valid criterion. I- $t$ curves of a 1 $\mathrm{MHz}$ double-potential step obtained with and without anthracene present in the solutions with $0.9 \mathrm{M} \mathrm{NEt}_{4} \mathrm{BF}_{4}$ as the supporting electrolyte are shown in Fig. 3A. From it we found that $i_{\mathrm{c}}$ decreased down to 0 in about $100 \mathrm{~ns}$, and $i_{\mathrm{f}}$ could be clearly discriminated, even at the end of the pulse. This indicated that the circuit could afford ultrafast DPSC for real electrochemical cells.

To judge the compensation level, $R_{\mathrm{c}}$ was adjusted to reach the optimized level, whose value was then determined to be $700 \Omega$. Because the optimized compensation level was $70 \%$, according to studies involving dummy cells, the solution resistance would be $10 \mathrm{k} \Omega$. It was greatly different from the theoretical value of $R_{\mathrm{s}}=\rho_{\mathrm{s}} / 4 r \approx 20 \mathrm{k} \Omega$, in which $\rho_{\mathrm{s}}$ of the applied solution was $20 \Omega$ $\mathrm{cm}{ }^{6}$ This great difference is due to simplifying the solution system to a pure $R C$ dummy cell. For a detailed discussion, refer to our previous paper. ${ }^{7}$

A simulated ${ }^{9} I-t$ curve is also presented in Fig. $3 \mathrm{~A}$, and the used parameters were as follows: $\alpha=0.45 ; k_{0}=5.1 \mathrm{~cm} \mathrm{~s}^{-1} ; D_{\mathrm{O}}=$ $D_{\mathrm{R}}=1.6 \times 10^{-5} \mathrm{~cm}^{2} \mathrm{~s}^{-1} ; E^{0}=-1.61 \mathrm{~V}$ versus the Pt reference (above values were employed by literatures); ${ }^{7,10}$ frequency $f=1$ $\mathrm{MHz}$; potential step $-1.1--2.2 \mathrm{~V} v s$. Pt electrode; $r=2.5 \mu \mathrm{m}$; $C=15 \mathrm{mM} ; R_{\mathrm{u}}=3 \mathrm{k} \Omega(70 \%$ compensation, optimized $) ; C_{\mathrm{d}}=$
$4.5 \mathrm{pF}$, which is a slightly greater than the former $3.8 \mathrm{pF}$, perhaps due to a greater stray capacitance caused by the more complexity of the circuit. A good fit between the experimental and simulated values demonstrated the validity of the instrumentation. Indeed, this kind of fit has been widely used to extract useful information, such as $k_{0}, D, \alpha$, and so on. ${ }^{1}$ A small bump appeared in the experimental curve, which may have come from impurities in the solution, such as traces of water and oxygen residues, but may also have resulted from an adsorption phenomenon of anthracene.

The current ratio $(R)$ is practically equal to the theoretical value of 1 , as shown in Fig. 3B. A higher performance function generator could further extend the lower limit of the time window, but this kind of extending is limited because a coupling of the diffusion layer and the double layer would occur, which means that the Frumkin's approximation should be reconsidered theoretically. ${ }^{10}$

\section{Conclusions}

In this work, ultrafast double potential step chronoamperometry at an ultramocroelectrode was achieved through online ohmic drop compensation, and its lower limit of the time window was extended to the submicrosecond level. Thus, this method will provide a useful tool to study faster kinetics and trace shorterlived intermediates. Early application of DPSC in kinetic studies has been summarized in detail for different mechanisms. ${ }^{1}$ A newer application is for calculation of dimerization rate constant $(k)$ according to the working curve $R$ vs. $k C \theta, 2,3,11,12$ in which $C$ is the concentration of the analyte.

\section{Acknowledgements}

This work was financed by a grant (No. 20173054) from the National Natural Science Foundation of China

\section{References}

1. A. J. Bard and L. R. Faulkner, "Electrochemical Methods: Fundmentals and Applications", 1980, John Wiley and Sons, New York, Chichester, Brisbane, Toronto.

2. C. P. Andrieux, P. Hapiot, and J. M. Savéant, J. Phys. Chem., 1988, 92, 5992.

3. C. Amatore, D. Garreau, M. Hammi, J. Pinson, and J. M. Savéant, J. Electroanal. Chem., 1985, 184, 1.

4. P. M. Narula and R. E. Noftle, J. Electroanal. Chem., 1999, 464, 123.

5. C. P. Andrieux, P. Hapiot, and J. M. Savéant, Chem. Rev., 1990, 90, 723 .

6. Z. X. Zhang, "Electrochemistry on Ultramicroelectrodes", 1998, Science Press, Beijing, 13.

7. Z. Y. Guo and X. Q. Lin, J. Electroanal. Chem., 2004, 568, 45.

8. C. P. Andrieux, D. Garreau, P. Hapiot, J. Pinson, and J. M. Savéant, J. Electroanal. Chem., 1988, 243, 321.

9. Z. X. Deng and X. Q. Lin, J. Electroanal. Chem., 1999. $464,215$.

10. C. Amatore, E. Maisonhaute, and G. Simonneau, J. Electroanal. Chem., 2000, 486, 141.

11. C. P. Andrieux, P. Hapiot, and J. M. Savéant, J. Am. Chem. Soc., 1987, 109, 3768.

12. B. M. Bezilla and J. T. Maloy, J. Electrochem. Soc., 1979, 126,579 . 